\title{
Study on Subjective Well-Being in Voluntourism of Females
}

\author{
Te-Yi Chang, Leo Huang, Shu Tang, Chia-Wen Chen \\ National Kaohsiung University of Hospitality and Tourism, Taiwan
}

\begin{abstract}
Along with the rise in modern feminine consciousness, many of the females would choose to leave out of their regular lives. They no longer aim at sightseeing or shopping in travel. Voluntourism has gradually become their priority to improve themselves, broaden their horizons, strength their wills, and get rid of inherent living circles. The purpose of this study is therefore to investigate the difference in female volunteer travelers in Taiwan. For this purpose, the study first starts from analyzing female background features, then their discrepancy in lifestyles, and based on the data collected, the authors also look into the difference of female involvement. Finally, the authors analyze the difference of their subjective well-being with the study result above. The study has collected a total of 98 valid samples focusing on females in Taiwan who had attended voluntourism through the Internet platform questionnaires. The study shows that female volunteer travelers are independent in lifestyles and prefer to interact with local people and other travelers. They are fond of arranging organized and flexible trips and they also have a strong interest in fashion trend and trendy events. The research shows that despite many of the female volunteer travelers are beginners in voluntourism, they generally present stronger subjective well-being. The result of the study is available for related departments to arrange and spread mid-term and long-term voluntourism for qualified and willing female volunteers with senior or experienced qualities. In the future literary planning, voluntourism can add on seeking happiness for more females who intend to realize their self-worth and pursue life meanings to understand the importance and meaning of voluntourism and enjoy in it.
\end{abstract}

Keywords: voluntourism, female, lifestyle, involvement, subjective well-being

\section{Introduction}

With the rise of modern feminist consciousness, many females in the Chinese society would not follow the ever unchanged life any more. Travel has gradually become a priority manner for them to cultivate themselves, exercise their willpower, expand their horizon, and get out of the routine life circle. There are always yearnings and expectations for life in their mind. They hope to know special persons and things during travels, to enjoy the unrestricted and unstressed life pleasure, and to learn more experience and knowledge. Women of different lifestyles are provided with different thinking manners but share the same ideas, which include especially how the females bound by old thoughts in the Chinese society break through themselves, acquire unexpected changes through the travel experience, and apply them in their life, work, and leisure.

Te-Yi Chang, Ph.D., Professor, Graduate Institute of Tourism Management, National Kaohsiung University of Hospitality and Tourism.

Leo Huang, Ph.D., Professor, Graduate Institute of Tourism Management, National Kaohsiung University of Hospitality and Tourism.

Shu Tang, Doctoral Student, Graduate Institute of Tourism Management, National Kaohsiung University of Hospitality and Tourism.

Chia-Wen Chen, Doctoral Student, Graduate Institute of Tourism Management, National Kaohsiung University of Hospitality and Tourism. 
Voluntourism that had its first origin in the early 1990s is flourishing in Asia presently. Some voluntourism activities and routes like the international volunteers have become increasingly favored by the youth (Benson, 2011). The emerging female voluntourism, not dominated by food and shopping any longer, awakens the special character "caring” of the female volunteer travelers, satisfies their psychological needs bit by bit, and brings them the spiritual contentment when they offer the volunteer service (McIntosh \& Zahra, 2007). Voluntourism has become a topic gaining more and more attention from the females as well as one of the new trends in the female tourism (Wearing \& McGehee, 2013).

With its impressive social, ecological, and economic benefits, voluntarism is favored and valued by the international market and the tourist destination countries (McGehee \& Andereck, 2009). Compared with its booming development, voluntourism still has not drawn much attention from the academic circle, and not enough research has been done about it. As far as the female voluntourism is concerned, there is even less discussion. Therefore, the purpose of this study is to investigate the difference in female volunteer travelers in Taiwan. For this purpose, the study starts from analyzing female background features and their discrepancy in lifestyles, looking into the difference of female involvement. Finally, the authors analyze the difference of their subjective well-being with the study result above.

Based on the background characteristics and lifestyles of Taiwanese female voluntourism, the research has studied their differences in lifestyles and involvement as well as the relations between lifestyles and involvement to underlie the research of voluntourism's social forms in the future and to provide the female voluntourism agents with a reference regarding the target market orientation, product design, and marketing strategy development.

\section{Literature Review}

\section{Female Backpacking}

According to Loker-Murphy and Pearce (1995), backpackers prefer the budget accommodations and traffic means, etc., value interaction with local people and other travelers, arrange independently the organized and flexible routes, travel longer than an ordinary vacation, and stress the informal rest.

Travel grows with the impulse of era development. With promotion in education and financial independence as well as delay of the marriage and childbearing age, modern women have gained higher autonomy in their life, owned more diversified information and resources, and taken active parts in backpacking (Tseng, 2000).

Although there is no related literature discussing the Taiwanese females travel in particular, multiple connections between travel and self-awareness have been much addressed. By interacting with different cultures, people, and things, travelers tender themselves in strange situations and increase the opportunities of self-growth and self-examination (Stratford, 2000). Researches both at home and abroad have concluded from analysis of backpackers' psychological motivations, desires, and needs that female backpackers feel more eager to explore the world and be more curious about different cultures and environments than the group travelers (Pritchard, 2001).

It has been increasingly the fashion for females to choose backpacking. They start to be unafraid of unknown dangers and difficulties in a journey, enjoy the surprises deriving from the journey, and expect to find an unknown self in this process. They deem backpacking a demonstration of another action power, another attitude to life, and a breakthrough to self-appeal of modern females (Holmes, Smith, \& Baum, 2010). The research tries to further explore the correlativity of specific drivers of backpacking with the social structure, mental motivations, personal values, and personality traits for modern Taiwanese women. 


\section{Voluntourism}

Voluntourism has a long history. It is a way of traveling started by the British non-profit organization National Trust Foundation. In voluntourism, the travelers bear their own transport and accommodations expenses; the service agent does not make payments but just offers simple meals (McGehee \& Santos, 2005; Wearing, 2004). The travelers do some volunteer services during the travel, including providing aids or supports to the impoverished or in environmental protection or social researches, so they make some active contributions to the travel destinations (Bussell \& Forbes, 2002; Wearing, 2002). In the broad sense, voluntourism is the experience acquired from the services in the destination to realize the aspiration of service. In the narrow sense, it is the experience combining organically the volunteer service with the traditional travel content including culture, geography, history, entertainment, etc. (Coghlan, 2006).

Voluntourism mentioned in this research refers to a special travel mode full of humanistic care, where travelers take part in activities featured with volunteer elements or dominated by volunteer service, increase their knowledge and experience, receive education, promote their comprehensive qualities, and offer help within their reach to improve the destination positively and promote its better development (Nguyen, 2012).

In the present literature, research of voluntourism basically focuses on the perspectives of its significance and values and rarely discusses the drivers and process experience on the part of females as well as changes brought by voluntourism to travelers. This paper, from the female perspective, has explored the field and made supplement to research in this field.

\section{Lifestyle}

The concept of lifestyle basically came from psychology and sociology. It is believed that every person has his or her special lifestyle. If common lifestyle perspectives of a group can be found, the group would be understood clearly and its behaviors can be predicted (Reynolds \& Darden, 1974).

Since the 1970s, the concept of lifestyle has been highly valued in the leisure consumption research, which is focused on the activities, interests, preferences, and opinions of consumers (Shih, Zheng, \& Chen, 2004).

According to Blackwell, Miniard, and Engel (2000), lifestyle is a systematic concept representing the life features demonstrated by the whole society or a consumer group. According to Hawkins, Best, and Coney (2001), lifestyle is about how we lead our life; it is a specific demonstration of ego-ideals and is shaped jointly by past experience, inner features, and situation of the time; it is strong enough to affect all levels of consumption behaviors.

To sum up, with lifestyle, we can know the ideas and preferences in consumers' mind as well as their consumption behaviors. If lifestyle is used as the benchmark for market segmentation, positions of different market segmentations of lifestyle in the society can be known after the market is segmented and the segmentations' correlation with demographic variables is analyzed.

Lifestyle mentioned in this paper refers to the daily life features and types of Taiwanese female volunteer travelers. It is used to understand the activity rules, interests, opinions, and other life manners of the group.

\section{Involvement}

Involvement refers to the degree of an individual's interest in a specific objective. According to Zaichkowsky (1985), involvement can be interpreted as the degree of one's attention on a certain thing based on his or her own needs, values, and interests. 
The leisure involvement proposed by Havitz and Dimanche (1999) refers to the awakened mental state when an individual experiences the recreation activities, tourist spots, or related recreation equipment and products and is characterized with the drivable behaviors.

Chuang, Lee, and Chung (2010) pointed out that involvement is subject to influences of internal psychology and external behavior factors including different individuals and situations and that different involvements would result in different behaviors and consumption decisions.

In the leisure and recreation research field, many scholars have adopted consumer involvement profile (CIP) to discuss the leisure and recreation behaviors. The CIP scale is essentially characterized with multiple perspectives. It is composed of four perspectives, which are described as follows (Havitz \& Dimanche, 1990):

(1) Importance: Importance of a product or activity perceived by an individual;

(2) Pleasure value: Involvement in leisure activities can satisfy an individual's need for pleasure value and is also an important index of his or her continuous participation and maintained interest in the leisure activities;

(3) Sign value: By taking part in leisure activities, an individual claims he or she is a member of a group or is distinguished from others; the higher involvement he or she is engaged in an activity, the better the activity represents his or her sign value;

(4) Perceived risk: The odds of subjective risks caused and wrong decisions made when an individual chooses and takes part in a leisure activity.

Involvement mentioned in this paper refers to the importance and care and engagement in voluntourism perceived by Taiwanese females. It is subject to influences of internal and external factors including different individuals and situations and causes influences on consumers' decisions. CIP, which comprises the four perspectives of importance, pleasure value, sign value, and perceived risk, has been used to measure the participation of Taiwanese female voluntourism.

To sum up, the research has discussed the importance, pleasure value, and risk perceived by Taiwanese females of different backgrounds and lifestyles in the voluntourism and their differences in subjective well-being with different involvements.

\section{Subjective Well-Being}

Research of the subjective well-being originated in 1970. A psychologist proposed that the element to constitute a nice life was that one feels himself or herself living a fairly good life. Such an orientation defining a nice life is called subjective well-being (Diener, 2000).

Subjective well-being proposed by Andrews and Withey (1976) is the overall assessment of an individual's subjective experience, including the life satisfaction as well as the perceived positive and negative emotion intensity (Chen, Gao, Han, Zhang, \& Li, 2016). In this research, the individual subjective cognition and emotion as well as life satisfaction, positive emotions, and negative emotions of Taiwanese females after completing voluntourism have been studied. Basically, the subjective well-being scale developed by Wu, Hsieh, and Wang (2006) has been applied. The scale measures the cognition part of subjective well-being of the respondents based on "The Satisfaction with Life Scale" (SWLS) by Diener, Emmons, Larsen, and Griffin (1985) and measures the emotional part of subjective well-being based on the collection of emotional terms designed by Diener, Smith, and Fujita (1995). The subjective well-being of Taiwanese females has been discussed based on SWLS and the positive and negative emotions scale. 
The research, taking the Taiwanese backpacking as the research target, has discussed their values and risks perceived in voluntourism with different background characteristics and lifestyles and discussed whether their life satisfaction and positive and negative emotions under the subjective well-being vary with different life experiences.

\section{Research Method}

The research has mainly discussed the relations between the lifestyles and the involvement variables of Taiwanese female volunteer travelers. Huang (1996) and Tseng (2000) found in the interviews with the female backpackers that their preparation before a travel, sights seen and new knowledge acquired by them during the travel had certain influences on their work or psychology after they come back from the travel. Thus, this research has included the complete process of female voluntourism before, during, and after the travel to increase the research's reliability.

Voluntourism defined in this research is a form of backpacking or travelling organized by a non-profit organization. In order to enrich the samples, the tour destinations (either domestic or overseas) as well as the number of tourists have not been limited. The research has been focused on selection of the voluntourism content types. The lasting days alone have been used as a reference for distinction between a short and long period and as a factor for studying the involvement. Thus, the research has just taken the Taiwanese females with the voluntourism experience as its samples.

During the research, Taiwanese females who had ever taken a voluntourism have been targeted to complete a questionnaire survey online. Based on the survey data, the background characteristics of Taiwanese female volunteer travelers, the significant differences in different perspectives of involvement by Taiwanese females with different lifestyles, and the correlation between their lifestyle and involvement have been analyzed and understood.

Samples for the research have been selected with the snowball sampling method. First, a list of females who have the voluntourism experience and travel websites, BBS and social communities, etc., have been acquired by organizing voluntourism as well as on related websites or forums. Then the females who have the voluntourism experience, irrespective of the participation times, a solo tour or a group tour, and a domestic or overseas destination, have been selected to answer the questionnaire online. The screening mechanism has been established in the first two questions in the questionnaire. The question about gender requires the answer "female", and the question "have you taken a voluntourism" requires the answer "yes". The respondents giving the said answers would be considered qualified for the basic conditions of a sample and allowed to answer the rest questions.

\section{Results}

The study period started on December 21, 2016 and ended on March 31, 2017. Altogether, 98 valid samples have been collected, based on which fundamental analysis has been carried out first.

\section{Background Characteristics}

Based on the above information, it can be known that people aged between 18-33 are the main group of voluntourism, accounting for $60 \%$, and the age range basically coincides with that of an undergraduate or postgraduate student. Female voluntourism older than 42 accounts for nearly 20\%, which indicates that respondents at all age groups are interested in voluntourism or are willing to take part in such travels dominated by volunteer service. 
According to survey results of marital status, the unmarried respondents account for nearly $60 \%$, which conforms to the age proportion. The married respondents with fertility account for nearly $40 \%$, indicating that many females with children are rather motivated to help others or accompany their children in related volunteer services to help them formulate right concepts and accumulate the volunteer service hours, which are required for their school promotion.

In respect of the education background, the respondents with the undergraduate education top the list by accounting for $70 \%$, since they have more opportunities to get access to voluntourism-related activities.

In respect of the average monthly salary, respondents with less than NTD 30,000 are the majority, because most of the respondents are students or fresh graduates. In combination with the occupational characteristics analysis, students and the service sector workers account for nearly $60 \%$, which is consistent with the above background data.

The most respondents take only one voluntourism on average annually, accounting for $57 \%$. In addition, the respondents who spend less than three days on a voluntourism make up $45 \%$. From these two results, it can be concluded that although most females feel like taking part in a voluntourism, there may be many restrictions, including the long travel time or the remote destination. Thus, most of them choose the voluntourism lasting less than a week and take part in the voluntourism for multiple times. Maybe this is a relatively new travel manner, and only those who are adventurous would probably take their part. The traveling partners are dominated by schoolmates or friends, who account for $70 \%$. Group travel may also be a reason for the small number of travelling days and times.

\section{Reliability Analysis}

Stability and consistency of the scales have been tested based on the Cronbach's $\alpha$. The value should be at least 0.6 and had better be higher than 0.7. The questionnaires for the research were released online, and 98 valid samples have been collected in Taiwan so far. Except the personal background, the questionnaire consists of four parts, namely the lifestyle scale, the involvement scale, the life satisfaction scale, and the positive and negative emotions scale. Followed are the reliability analysis results of the scales.

The values of Cronbach's $\alpha$ of the lifestyle scale are as follows: family orientation perspective: 0.67 ; comprehensible experience perspective: 0.71 ; fashion perspective: 0.63 ; reality breakaway perspective: 0.63 ; total scale: 0.86 . They indicate the high consistency between the test perspective questions that are able to test the perspective concepts completely.

The values of Cronbach's $\alpha$ of the involvement scale are as follows: perceived value perspective: 0.86 ; perceived risk perspective: 0.53 ; total scale: 0.85 . They indicate medium to high consistency between the test perspective questions that are able to test the perspective concepts almost completely.

The value of Cronbach's $\alpha$ of the total subjective well-being, life satisfaction scale, is 0.85 . It indicates high consistency between the test perspective questions that are able to test the perspective concepts completely.

The values of Cronbach's $\alpha$ of the subjective well-being, positive and negative emotions scale, are as follows: the positive emotions perspective: 0.60 ; the negative emotions perspective: 0.60 ; the total scale: 0.74 . They indicate the acceptable consistency between the test perspective questions that are able to test the perspective concepts almost completely. 


\section{Results for Lifestyle}

From the family orientation perspective, we can see that about half of the respondents say no to the questions about "festival reunion", "photo collection”, and "holiday reunion”, which indicates that the females feel good to part with the family for a period and are relatively independent. In addition, regarding "shopping around" and "expenses recording", the number of respondents who agree is almost the same with that of the respondents who disagree, which indicates that the local living environment would also affect the females' consumption during a voluntourism. Finally, more than half of the respondents disagree with "buying voluntourism souvenirs for friends", probably because the souvenirs in voluntourism destinations are generally simple and rough or the purpose of the travel is volunteer service rather than sightseeing. Thus, they usually do not buy souvenirs for their friends.

From the comprehensible experience perspective, it can be seen that about half of the respondents disagree to "know about history and culture", "visit local schools", and "challenging and competitive activities", which indicates that the females are concentrated on the volunteer service itself rather than other sightseeing activities. In addition, regarding "sports events live broadcast", the number of respondents who agree is almost the same with that of the respondents who disagree, which indicates the females are active in the daily life and most of them like social activities. Finally, the majority of respondents agree to "active in social activities", which indicates that the females like to take part in different social activities, blend in a group, and play an active role there.

From the fashion perspective, it can be seen that half of the respondents disagree to "making the schedule and completing the plan”, which indicates that most females take a voluntourism on the spur of the moment, do not plan it as a long-term activity, and do not care much about the travel itinerary. In addition, more respondents agree to "being dressed in fashion”, "loving fashion”, and "preferring the venues where popular events come about", which indicates that most females are young at heart, fashion-conscious, and care about both current and hot events. It can be concluded that they also take voluntourism as a popular, fashionable, and novel travel manner.

From the reality breakaway perspective, more respondents disagree to "being far away from the usual living environment" and "choosing a destination far away from the hubbub", which indicates that most females do not take their part passively out of the living pressure but because of their enthusiasm about "care" and "service".

\section{Results for Involvement}

From the perceived value perspective, it can be seen that more respondents disagree to "meaning", "interest”, “joy”, “comprehension”, and "help”. It is speculated that most females in this survey are freshmen of voluntourism, so they do not have enough experience to develop an interest or habit and do not perceive the effect from permanent support of volunteer service. After all, the effect brought by voluntourism does not come about immediately, but needs time and space for accumulation.

From the perceived risk perspective, regarding "unsure about whether it is safe" and "inappropriate voluntourism would have serious results", the number of respondents who agree is almost the same with that of the respondents who disagree, which indicates that females would pay attention to risks of a voluntourism before or after it and hope to reduce the odds of making mistakes. 


\section{Results for Subjective Well-Being}

From the life satisfaction perspective, it can be seen that more respondents agree to "be close to the ideal", "live a good life", and "feel satisfied", which indicates that the females feel satisfied about their personal life status and believe it is close to their ideal state. In addition, regarding "already acquire the important things in the life", the number of respondents who agree is almost the same with that of the respondents who disagree, which indicates that the females still fight actively for the important things in their life, being either the material or spiritual satisfaction, or the life enrichment.

From the positive emotions perspective, more respondents always feel "joyful”, "pleased", and "happy", which indicates that most females have the optimistic and positive emotions and enjoy the higher subjective well-being, so they also deliver enough positive energy to people who need care and help in the voluntourism.

From the negative emotions perspective, more than half of the respondents feel occasionally "be cared about", "angry", "sad", and "unhappy", which indicates that females would get the negative emotions occasionally, but the emotions do not affect their subjective well-being significantly.

\section{Discussion}

Based on the purpose and the empirical survey and analysis, the research has discussed the conclusions, so as to know about the background characteristics, lifestyles, involvement, and subjective well-being as well as the relations among Taiwanese female volunteer travelers. Suggestions for the future research have been made as well. The research results would be provided to related administrations as a reference to help public service organizations and individuals reach double win.

Respondents aged between 18 and 25 make up the largest percentage of 35.7\%, and respondents between 26 and 33 make up $24.5 \%$, which indicates that the female volunteer travelers are dominated by young people. Respondents who are unmarried account for the largest percentage of $58.1 \%$, indicating that most females are unmarried. Respondents whose education level is identified as "college or university" make up the largest percentage of $70.5 \%$, indicating that most females have got the college or university education. Regarding the occupational status, "students" account for the largest percentage of $36.7 \%$, followed by the "service sector" with $19.4 \%$, indicating that the occupational distribution of females is concentrated on some specific groups.

Respondents who earn less than NTD 22,000 on average every month make up the largest percentage of $41.8 \%$, followed by those who earn NTD 22,001-30,000 with 20.4\%, indicating that the females have the medium-low income. Respondents taking only one voluntourism on average annually make up the largest percentage of $57.1 \%$, followed by those taking from two to four times with $39.8 \%$, indicating the slightly low frequency of Taiwanese females' participation in volunteer service. Respondents spending less than three days on a voluntourism on average make up the largest percentage of $45.9 \%$, followed by those spending from two to seven days with $35.8 \%$, indicating that the volunteer service activities of Taiwanese females are dominated by the short-term tour. The traveling partners in a voluntourism are dominated by "schoolmates/friends", who account for the largest percentage of $75.4 \%$, indicating that females go on a voluntourism most often with their schoolmates or friends.

Voluntourism is a new trend in recent years. To young people, it is one of the popular events. By taking part in a voluntourism, they can not only be engaged in the fashion, but also show that they are glad to offer help and satisfy their self-accomplishment. Also, it demonstrates their trait of loving fashion. 
A female volunteer traveler may last for a week, a month, and even over half a year. If one takes on a job with fixed working hours, it would be hard for him or her to give up the work and take a voluntourism. According to the research results, most females are students or service sector workers. Since students do not get employed, they can give themselves a precious opportunity for service and for taste of novel experience. The service sector workers have the high turnover rate, so they are able to take part in activities easily. The students and the service sectors share a common trait. Students or freshmen at work need something to make them feel needed. Thus, voluntourism is not only a trend, but also a spiritual satisfaction. The unmarried status also gives them great flexibility in using time. This is also one of the traits of the young females who have the flexible jobs.

The survey samples are dominated by students. Although they do not find the important things in their life during the females or do not feel the substantial meaning or value brought about by voluntourism, they feel joy in their life and adjust their consumption behaviors in the process of voluntourism.

According to the research results, most young people have the positive emotions and are willing to try a voluntourism. Although they are willing to take part in such activities, they may still feel unsecured about voluntourism, as they do not develop a plan, have no traveling partners, do not think well before making the decision, become doubtful easily, or get worried by their family. In particular, such a feeling of insecurity is more obvious when they take part in a voluntourism overseas.

\section{Conclusion}

Based on the statistics of females with different background characteristics, most females are young and have acquired the college education or above. Meanwhile, most of them are students or service sector workers. They do not have a high income. The majority of them take a voluntourism with their schoolmates or friends. Their voluntourism usually lasts for less than a week, and they usually take only one voluntourism annually.

It is suggested that inter-college cooperation or winter/summer holiday voluntourism be carried out for students, so more enthusiastic college students can take part in the volunteer service activities and have a novel and meaningful travel during the holiday.

Based on the statistics of females with different lifestyles, it has been found that the Taiwanese female volunteer travelers lead an independent life, have the rational family concept, prefer the economic accommodations, transport means and facilities, pay attention to interaction with local people and traveling partners, like to arrange the organized and flexible itinerary themselves, pay close attention to fashion news, and have much interest in hot events and venues.

Based on the statistics of females with different involvements, most Taiwanese females spend a short time on a voluntourism and do not travel frequently. They are fresh, so they do not have enough experience accumulated to develop an interest or habit, and do not perceive the effect brought by permanent support of public service activities. It is suggested related administrations arrange the medium-long voluntourism for the intermediate travelers who are willing and qualified to take part in such. After all, effect brought by voluntourism does not come about immediately. It needs time and space for accumulation.

Based on the statistics, Taiwanese females have strong subjective well-being. In modern society, females have the independent characters and other traits, keep promoting their education level and social position, delay the marriage and childbearing time, and become independent financially. Such a phenomenon benefits their ability to take a voluntourism and stick out more the traits and self-growth opportunities of females. 
From the perspective of subjective well-being, the happiness-seeking elements can be added in the future travel route planning, so more Taiwanese females that are trying hard to realize their self-worth and seek the significance of life would become aware of the meaning and value of voluntourism, take part in voluntourism, and promote their well-being as most participants do.

\section{References}

Andrews, F. M., \& Withey, S. B. (1976). Social indicators of well-being. New York, NY: Plenum Press.

Benson, M. A. (2011). Volunteer tourism: Theory framework to practical applications. New York, NY: Routledge.

Blackwell, R. D., Miniard, P. W., \& Engel, J. F. (2000). Consumer behavior (9th ed.). London: Harcourt Brace College.

Bussell, H., \& Forbes, D. (2002). Understanding the volunteer market: The what, where, who and why of volunteering. International Journal of Nonprofit and Voluntary Sector Marketing, 7(3), 244-257.

Chen, R. S., Gao, X. F., Han, P. L., Zhang, X. Y., \& Li, Y. H. (2016). Happiness: Positive psychology. New Taipei City: National Open University.

Chuang, J. C., Lee, C. P., \& Chung, C. C. (2010). A study on relationship among tourism image, tourism experience and behavioral intentions-A case study of Gukeng tourist area. Leisure Sports Journal, 9, 47-59.

Coghlan, A. (2006). Volunteer tourism as an emerging trend or an expansion of ecotourism? A look at potential clients' perceptions of volunteer tourism organizations. International Journal of Nonprofit and Voluntary Sector Marketing, 11(3), 225-237.

Diener, E. (2000). Subjective well-being. The science of happiness and a proposal for a national index. American Psychologist, 55(1), 34-43.

Diener, E., Emmons, R. A., Larsen, R. J., \& Griffin, S. (1985). The satisfaction with life scale. Journal of Personality Assessment, 49(1), 71-75.

Diener, E., Smith, H., \& Fujita, F. (1995). The personality structure of affect. Journal of Personality and Social Psychology, 69(1), 130-141.

Havitz, M. E., \& Dimanche, F. (1990). Propositions for testing the involvement construct in recreational and tourism contexts. Leisure Sciences, 12(2), 179-195.

Havitz, M. E., \& Dimanche, F. (1999). Leisure involvement revisited: Drive properties and paradoxes. Journal of Leisure Research, 31(2), 122-149.

Hawkins, D. I., Best, R. J., \& Coney, K. A. (2001). Customer behavior: Building marketing strategies (8th ed.). Boston: Irwin/McGraw-Hill.

Holmes, K., Smith, K., \& Baum, T. (2010). Volunteers and volunteering in leisure: Social science perspectives. Leisure Studies, 29(4), 435-455.

Huang, C. F. (1996). Backpacking as a construction of authentic experience - A caseese female backpacker to Europe. Taichung: Tunghai University.

Loker-Murphy, L., \& Pearce, P. (1995). Young budget travelers: Backpackers in Australia. Annals of Tourism Research, 22(4), 819-843.

McGehee, N. G., \& Santos, C. A. (2005). Social change, discourse and volunteer tourism. Annals of Tourism Research, 32(3), 760-779.

McGehee, N., \& Andereck, K. (2009). Volunteer tourism and the "voluntoured": The case of Tijuana, Mexico. Journal of Sustainable Tourism, 17(1), 39-51.

McIntosh, A. J., \& Zahra, A. (2007). A cultural encounter through volunteer tourism: Towards the ideals of sustainable tourism. Journal of Sustainable Tourism, 15(5), 541-556.

Nguyen, L. M. (2012). Volunteer tourism program development, case Vietnam heritage travel. Finland: Rovaniemi University of Applied Sciences.

Pritchard, A. (2001). Tourism and representation: A scale for measuring gendered portrayals. Leisure Studies, 20(2), 79-94.

Reynolds, F. D., \& Darden, W. R. (1974). Constructing life style and psychographics. In W. D. Wells (Ed.), Life style and psychographics. Chicago: American Marketing Association.

Shih, J. F., Zheng, C. H., \& Chen, Y. Y. (2004). Comparison on the customer behaviors of resort hotel in between Kending and Chihpen. Tourism Management Research, 4, 67-80. 
Stratford, E. (2000). Gender, place and travel: The case of Elsie bikes, South Australian pioneer. Journal of Australian Studies, 66, 116-131.

Tseng, H. M. (2000). A study of traveling experience of women individual travelers. Taipei: National Taiwan University.

Weaning, S. (2002). Volunteer tourism: Experiences that make a difference. Wallingford: CABI Publishing.

Wearing, S. (2004). Examining best practice in volunteer tourism. In R. A. Stebbins and M. Graham (Eds.), Volunteering as leisure/leisure as volunteering: An international assessment. Wallingford: CABI Publishing.

Wearing, S., \& McGehee, N. G. (2013). Volunteer tourism: A review. Tourism Management, 38, 120-130.

Wu, C. C., Hsieh, C. M., \& Wang, W. (2006). Construction and examination of structural equation model of leisure participation, leisure satisfaction and subjective well being. Leisure Sports Journal, 5(4), 153-168.

Zaichkowsky, J. L. (1985). Measuring the involvement construct. Journal of Consumer Research, 12(3), 341-352. 